\title{
Text-Enriched Representations for News Image Classification
}

\author{
Elias Moons \\ Department of Computer Science \\ KU Leuven \\ elias.moons@cs.kuleuven.be
}

\author{
Tinne Tuytelaars \\ Department of Electrical Engineering \\ KU Leuven \\ tinne.tuytelaars@kuleuven.be
}

\author{
Marie-Francine Moens \\ Department of Computer Science \\ KU Leuven \\ sien.moens@kuleuven.be
}

\begin{abstract}
Images have a prominent role in the communication of news on the Web. We propose a novel method for image classification with subject categories when limited annotated images are available for training the classifier. A neural network based encoder learns image representations from paired news images and their texts. Once trained, this encoder transforms any image to a text-enriched representation of the image, which is then used as input for the classifier that categorizes an image according to its subject category. We have trained classifiers with different amounts of annotated images and found that the image classifier that uses the text-enriched image representations outperforms a baseline model that only uses image features especially in cases with limited training examples.
\end{abstract}

\section{INTRODUCTION}

Web news companies receive a lot of images that could be used in news articles, but these are often not accompanied by text and even less by subject categories. For instance, on social media users post large amounts of images of local news that could be picked up by journalists. We need technology that classifies and filters these images into subject categories. The constraints are that within-class variability of news image patterns is huge and the number of annotated training data is limited. Automated classification of Web content has a long-standing tradition, but is mostly restricted to the classification of textual documents where SVM's form an established technique. The availability of large scale benchmark datasets such as ImageNet [1] has boosted image classification based on deep learning. Classifiers trained on these benchmarks are used directly to classify Web images/video (e.g. [4]). They are also used as a way to obtain a powerful image representation on top of which a final classifier can be trained [5], or as initialization of (part of) another neural network (finetuning) [9]. Such transfer learning techniques have drastically reduced the amount of application-specific annotations needed compared to training models from scratch, but are still insufficient for dealing with categories with large within-class variability. There are some works that integrate context when classifying Web visual content. Examples are the work of Guermazi et al. [3] or Song et al. [7], who exploit textual descriptions that accompany images or video. These methods need images and their context information as input for the classifier, while we want to build a subject classifier that uses solely images as input. Various unsupervised image representations based on spatial or temporal

This paper is published under the Creative Commons Attribution 4.0 International (CC BY 4.0) license. Authors reserve their rights to disseminate the work on their personal and corporate Web sites with the appropriate attribution.

WWW' 18 Companion, April 23-27, 2018, Lyon, France

() 2018 IW3C2 (International World Wide Web Conference Committee), published under Creative Commons CC BY 4.0 License.

ACM ISBN 978-1-4503-5640-4/18/04.

https://doi.org/10.1145/3184558.3186948 (e.g., [8]) structural information have been proposed, which proved to reduce the number of training data in visual classification. We follow a similar philosophy, but instead we propose to enrich news image representations or embeddings with textual information. We propose a neural encoder which is trained on the large image-text dataset (i.e., news articles with text and images) and which at test time builds a text-enriched representation of any input image. The actual subject neural classifier is trained on labeled image data. It takes the text-enriched image representation as input and produces membership predictions for all subject classes. The contribution is a novel news image classifier that uses the text-enriched image representations, which proves to be advantageous compared to a traditional image classifier which only uses image features.

\section{METHODS}

\subsection{Text-enriched image classifier}

2.1.1 Encoder. The input of the encoder is an image vector representation $x^{e n c}$ of a training example. This vector passes through two fully connected layers with transformation matrices $W^{e n c, 1}$ and $\boldsymbol{W}^{e n c, 2}$, obtaining respectively a hidden representation $\boldsymbol{h}^{e n c}$ and an output $\boldsymbol{y}^{e n c}$. The state of one hidden node $h_{i}^{e n c} \in \boldsymbol{h}^{e n c}$ is calculated as follows (with $F$ being a softmax activation function):

$$
h_{i}^{e n c}=\sum_{j=1}^{m} F\left(w_{i, j}^{e n c, 1} \cdot x_{j}^{e n c}\right)
$$

with $m=4096$. The state of an output node $y_{i}^{e n c} \in y^{e n c}$ becomes:

$$
y_{i}^{e n c}=\sum_{j=1}^{n} F\left(w_{i, j}^{e n c, 2} \cdot h_{j}^{e n c}\right)
$$

with $n=1024$. At training time, the encoder learns to predict the corresponding text representation for a given image (both 4096dimensional vectors). At test time, it produces a corresponding text-enriched image representation for a given image.

2.1.2 Classifier. The input of the classifier is an enriched image vector representation $x^{e n r}$. This vector passes through two fully connected layers with transformation matrices $\boldsymbol{W}^{e n r, 1}$ and $\boldsymbol{W}^{e n r, 2}$, obtaining respectively a hidden representation $\boldsymbol{h}^{e n r}$ and an output $y^{e n r}$. The state of one hidden node $h_{i}^{e n r} \in \boldsymbol{h}^{e n r}$ is calculated as:

$$
h_{i}^{e n r}=\sum_{j=1}^{o} F\left(w_{i, j}^{e n r, 1} \cdot x_{j}^{e n r}\right)
$$

with $o=4096$. The state of an output node $y_{i}^{e n r} \in y^{e n r}$ becomes:

$$
y_{i}^{e n r}=\sum_{j=1}^{p} F\left(w_{i, j}^{e n r, 2} \cdot h_{j}^{e n r}\right)
$$


with $p=256$. For a given (enriched) image (representation), the output of the network will be a vector with membership predictions for each of the subject categories. During training the two networks are considered separately. The encoder is trained on the news image-text dataset. The image classifier is trained only on annotated images. At test time an image is subsequently sent through the encoder and then through the image classifier to obtain subject category membership predictions.

\subsection{Image-only baseline}

The architecture of the traditional image-only model is identical to the image classifier described above. This model is trained to predict memberships for the different classes solely based on the image features without first sending these features through an encoder.

\section{EXPERIMENTS AND RESULTS}

\subsection{Data}

The data used to evaluate the models comes from the Webhose ${ }^{1}$ dataset, which classifies news articles into 7 subject categories (entertainment, finance, politics, sports, technology, travel and world news) defining a multiclass and multilabel categorization. A preprocessing step is shared between the proposed model and the baseline. An image is described by 4096 features generated by a VGG-16 network, a deep convolutional network [6]. To train the encoder, a text is represented as a bag-of-words using the 4096 most informative words calculated by the Chi-Square statistic [2] on a labeled text dataset. A test set of 500 samples is drawn uniformly at random from the Webhose dataset keeping only the images and their ground-truth subject labels and making sure that its images do not occur in the rest of the dataset, leaving an actual test set of 378 samples. The training data for the encoder (7384 images and their co-occurring texts) and the training data for the image classifier (various sizes, images with their classification label) are obtained from the remainder of the Webhose dataset. ${ }^{2}$ In all experiments $20 \%$ of the training data is used for validation and parameter tuning. An output score threshold of 0.5 is used for category prediction. We report micro-averaged precision, recall and F1 scores.

\subsection{Training with limited annotated images}

For all multiples of 1000 smaller than the size of the image classifier training set, training sets are selected uniformly at random of that specific size. This experiment was repeated 5 times in total and the scores are averaged over all the experiments to obtain more qualitative results. The F1 scores in Figure 1 show that using text-enriched image representations in news image classification outperforms the image-only baseline classifier for all considered sizes of the training data. The performance gain of the text-enriched classifier is more prominent when the annotated training set is smaller. Table 1 gives detailed results for a dataset of 2000 and 5000 annotated training images. In case of only 2000 training images, the text-enriched classifier improves the baseline by $4.93 \%$ (p-value of $0.023,2$-tailed $\mathrm{t}$-test). For the case of 5000 samples the difference in F1 score is $1.84 \%$ but still significant ( $\mathrm{p}$-value of 0.040 ).

\footnotetext{
${ }^{1}$ https://webhose.io/datasets.

${ }^{2}$ Data splits can be found at: http://liir.cs.kuleuven.be/software.php.
}

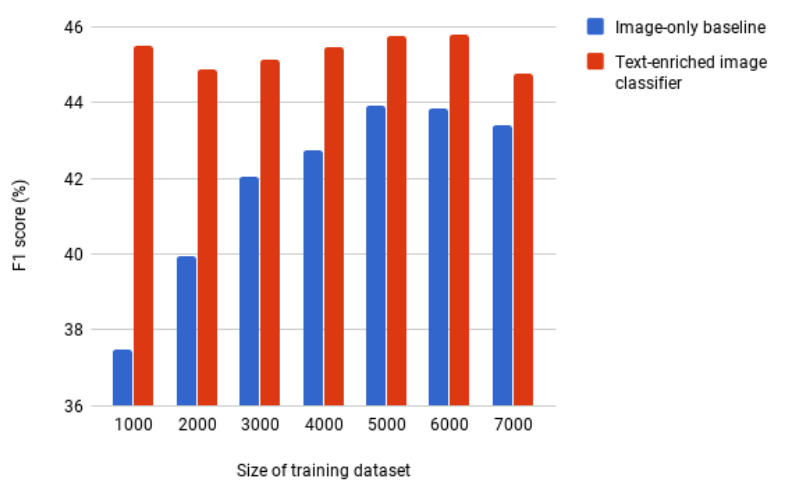

Figure 1: F1 score of the image classifiers in function of the fraction of the annotated image dataset.

Table 1: Accuracy, precision, recall and F1 scores for the image classification models trained on 2000 and 5000 annotated training instances

\begin{tabular}{l|c|cccc}
\hline Model & Size & Acc & Prec & Rec & F1 \\
\hline \hline Image & 2000 & $87.18 \%$ & $60.47 \%$ & $29.84 \%$ & $39.93 \%$ \\
Text-enriched & 2000 & $87.21 \%$ & $58.34 \%$ & $36.46 \%$ & $\mathbf{4 4 . 8 6 \%}$ \\
\hline Image & 5000 & $87.41 \%$ & $60.48 \%$ & $34.55 \%$ & $43.92 \%$ \\
Text-enriched & 5000 & $87.10 \%$ & $57.34 \%$ & $38.10 \%$ & $\mathbf{4 5 . 7 6 \%}$ \\
\hline
\end{tabular}

\section{CONCLUSION}

The proposed text-enriched image classifier outperforms a purely image-feature based classifier on a Web news image dataset, which is characterized by a large variety of visual patterns and limited labeled training data.

\section{ACKNOWLEDGMENTS}

The research was funded by the Google DNI grant MetaHaven.

\section{REFERENCES}

[1] Jia Deng, Wei Dong, Richard Socher, Li-Jia Li, Kai Li, and Li Fei-Fei. 2009. ImageNet: A large-scale hierarchical image database. In CVPR 2009. IEEE, 248-255.

[2] Persi Diaconis and Bradley Efron. 1985. Testing for independence in a two-way table: New interpretations of the chi-square statistic. The Annals of Statistics 13, 3 , 845-874. http://www.jstor.org/stable/2241103

[3] Radhouane Guermazi, Mohamed Hammami, and Abdelmajid Ben Hamadou. 2010. Classification of violent Web images using context based analysis. In SAC, Sierre, Switzerland, March 22-26, 2010. 1768-1773.

[4] Tao Mei and Cha Zhang. 2017. Deep learning for intelligent video analysis. In MM, Mountain View, CA, USA, October 23-27, 2017. 1955-1956. https://doi.org/10. 1145/3123266.3130141

[5] Ali Sharif Razavian, Hossein Azizpour, Josephine Sullivan, and Stefan Carlsson. 2014. CNN features off-the-shelf: an astounding baseline for recognition. In CVPR. 806-813.

[6] Karen Simonyan and Andrew Zisserman. 2014. Very deep convolutional networks for large-scale image recognition. CoRR abs/1409.1556. arXiv:1409.1556

[7] Yang Song, Ming Zhao, Jay Yagnik, and Xiaoyun Wu. 2010. Taxonomic classification for web-based videos. In CVPR, 2010. IEEE, 871-878.

[8] Xiaolong Wang and Abhinav Gupta. 2015. Unsupervised learning of visual representations using videos. In ICCV. 2794-2802.

[9] Jason Yosinski, Jeff Clune, Yoshua Bengio, and Hod Lipson. 2014. How transferable are features in deep neural networks?. In NIPS. 3320-3328. 